\title{
COMPUTATIONAL MODELING OF THE DYNAMIC BEHAVIOR OF THE INDUCTION MOTOR ROTOR
}

\author{
DONAT, M[artin]
}

\begin{abstract}
This paper presents a process of calculating of the dynamic response of the cage induction motor with eccentric rotor, which is excited by unbalanced magnetic pull. The unbalanced magnetic pull, acting on the rotor, was obtained from the results of the electromagnetic coupled field analysis as function of the air gap eccentricity and rotation angle of the rotor bars due to the stator winding slots. The dynamic response of the rotor, excited by the unbalanced magnetic pull, was calculated by finite element time-stepping analysis in Ansys software. The modeling is done for the case when the rotor is parallel due to stator. The obtained results are presented as the trajectory of the shaft center.
\end{abstract}

Keywords: cage induction motor, rotor, stator, unbalanced magnetic pull

\section{INTRODUCTION}

Magnetic fields, operating in the air gap of the rotating electrical machines, induce electromagnetic forces which act on the machine structure. Ideally, the air gap thickness is constant along a whole circumference and the electromagnetic forces cancel out each other. In fact, the air gap thickness is not constant along a whole circumference, for example due to eccentrically mounted rotor with respect to the stator, shape deviations of the rotor and the stator from ideal cylindrical shape or dynamic eccentricity. The air gap thickness is not constant along a whole circumference in these cases, electromagnetic forces do not cancel out each other and the resulting electromagnetic force is called as the unbalanced magnetic pull (UMP). The UMP is composed of the radial and tangential component. The radial component of UMP is usually significantly larger than tangential one; therefore the UMP acts approximately in direction of minimal air gap thickness and its magnitude depends on the air gap asymmetry and rotation angle of the rotor bars due to the stator winding slots.

In the last decade, several studies of the influence of UMP on the dynamic behavior of the rotors of the rotating electrical machines have been published. For example, publications [1] and [2] used to study the influence of UMP on vibration of the rotor of the rotating electric machine computational models, which are a combination of the analytical computational model of UMP and de Laval model of the rotor. The amplitude of UMP is, in fact, affected by nonlinear saturation effect of the stator and rotor winding core; therefore the simple parametric computational model of UMP, which include this effect, is used with the de Laval model of the rotor to study the influence of UMP on stability of the rotor motion in [3]. The computational model of UMP used in this study is not purely analytical, because the parameters of this computational model are determined from results of electromagnetic forces, acting on the rotor, which were calculated by finite element method. Since, the rotors of the rotating electrical machines are not absolutely stiff and the dynamic displacement of the rotor is not constant along the rotor axis the finite element computational model of the rotor is used with combination of the analytical computational model of the UMP to investigate the influence of UMP on the dynamic behavior of the generator in [4].

The experimental investigation of the influence of UMP on the dynamic behavior of the rotors of the rotating electrical machines is difficult and too expensive; hence the computational modeling is used. Analytical computational models of UMP have been used in general. Nevertheless, it is difficult to include in these computational models some phenomena that can significantly affect the magnitude of UMP, for example the effect of slot harmonics and saturation effect of the ferromagnetic core of rotor and stator windings. This applies to all the above mentioned besides [3].

This paper presents a process of computational modeling of the dynamic behavior of the rotor of the rotating electrical machine in commercial finite element software Ansys. The calculation is performed in two phases. In the first phase, dependence of UMP on the air gap asymmetry and rotation angle of the rotor bars with respect to the stator windings slots is obtained and this dependence is used in the second phase, which deals with the study of forced vibrations caused by UMP.

\section{METHODS}

Both phases of the calculation were performed in the commercial finite element software Ansys.

\subsection{Calculation of UMP}

UMP is the resultant of electromagnetic forces acting on the rotor of the machine. Computational model of UMP, used in this paper, is described in detail in [5]. This computational model of UMP was composed of the two-dimensional computational model of the magnetic circuit of the machine and the computational model of the electric circuit of the machine. Both computational models are coupled together and solved simultaneously by finite element method. Nonlinear magnetic properties of the stator and the rotor winding core were described by $\mathrm{B}-\mathrm{H}$ curve to include saturation effect into the computational model. Normal and tangential components of UMP, acting on the rotor, were calculated from results of the nonlinear harmonic analysis by Maxwell stress tensor method for different values of air gap asymmetry. Due to consider the slot harmonics effect, normal and 


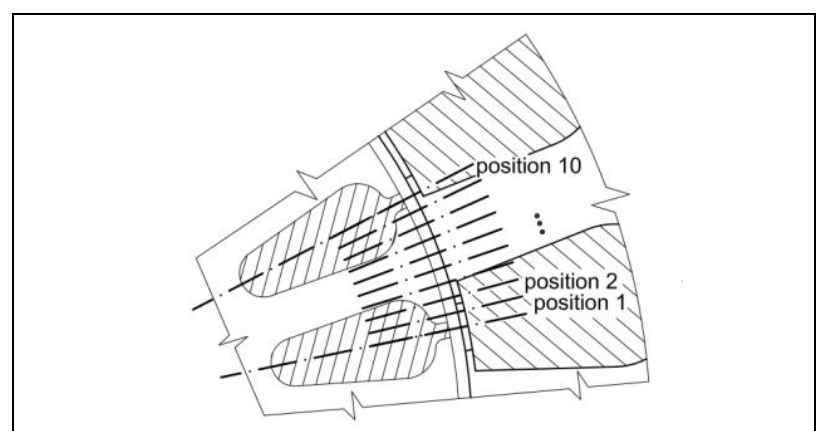

Fig. 1. Positions of the rotor bars

tangential components of UMP were calculated in ten different positions of the rotor bars with respect to the stator winding slots for each value of the air gap asymmetry, as shown Figure 1. Dependence of normal and tangential components of UMP on the air gap asymmetry and rotation angle of the rotor bars due to stator winding slots were obtained from performed analysis and these dependences were used in the next step to study the influence of UMP on the dynamic behavior the rotor of the machine.

\subsection{Calculation of the dynamic response of the rotor}

The dynamic response of the rotor excited by UMP was calculated by finite element time-stepping analysis. The computational model of the rotor is created by beam elements BEAM188. This rotor is supported by two ball bearings. The stiffness of these supports is nonlinear function of the load, as shown Figure 2; hence they are modeled by nonlinear spring elements COMBIN39. It is assumed that the frame of the machine is absolutely stiff, this frame is not include in the computational model; therefore zero displacements were prescribed to nodes at the free ends of the elements COMBIN39. Moreover, support $\mathrm{B}$ inhibits the motion of the rotor in the axial direction, hence zero displacement in z-direction was prescribed to the node 0 too, see Figure 3. The stiffness of the rotor winding is not known; therefore it is assumed that the material of the rotor is homogeneous linearly elastic and the material properties of the rotor were described by Young's modulus, Poisson's ratio and density of the material. Young's modulus and Poisson's ratio were entered as the whole rotor was made of iron. The density of the material of the rotor was determined from mass $m_{r}$ and volume $V_{r}$ of the rotor by (1).

$$
\rho_{r}=\frac{m_{r}}{V_{r}}
$$

The UMP is continuously distributed load along the rotor winding axis in the real rotating electric machine; therefore the computational model of the rotor was divided into several segments. The UMP, acting on segments, was modeled by prescribing $x$ and $y$ components of UMP to the centers of gravity of these segments, as shown Figure 3, subscripts I, II and III denote number of segment.

The time-stepping analysis means that the dynamic response of the rotor to the UMP was calculated by transient analysis at discrete time steps. The time step length was set to correspond of rotation of the rotor of $0.3^{\circ}$ and the UMP is constant during this time step. The dependence of normal and tangential components of UMP on the air gap asymmetry and rotation angle of the rotor bars due to stator winding slots were included in this computational model by two two-dimensional arrays. Columns of these arrays describe dependence of normal and tangential components of UMP on the air gap asymmetry, rows of these arrays describe dependence of normal and tangential components on the rotation angle of the rotor bars due to stator windings slots. The total displacements $x_{r}$ and $y_{r}$ of the segments centers of gravity were evalued in direction $\mathrm{x}$ and $\mathrm{y}$ at the end of each time step. These total displacements include dynamic displacements of the rotor caused by UMP and static displacements caused by eccentric mounting of the rotor due to stator. Magnitude of minimal air gap thickness in radial direction was determined from these displacements for each rotor winding segment. The normal and the tangential components of UMP were determined for the next time step from the created twodimensional arrays by linear interpolation on the grounds of minimal air gap thickness and rotation angle of the rotor bars due to stator winding slots and these components were transferred to the $\mathrm{x}$ and $\mathrm{y}$ directions of global Cartesian coordinate system. This process describes (2) and (3) and Figure 4.

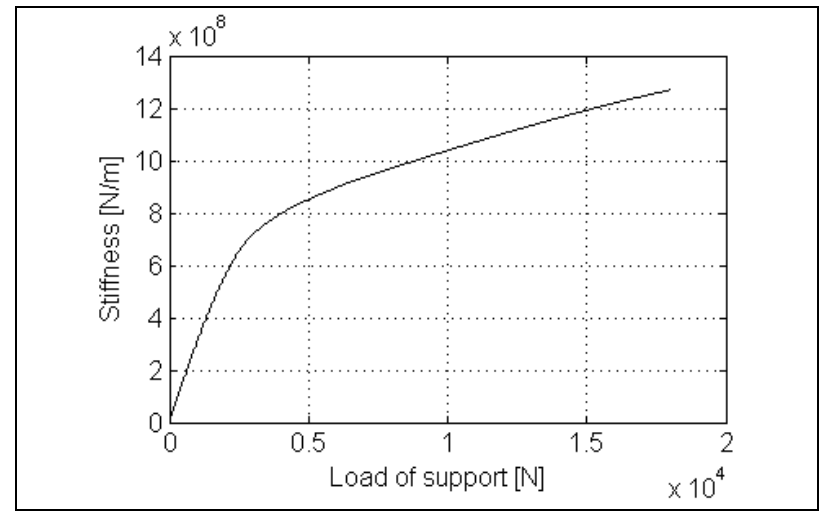

Fig. 2. Stiffness of support

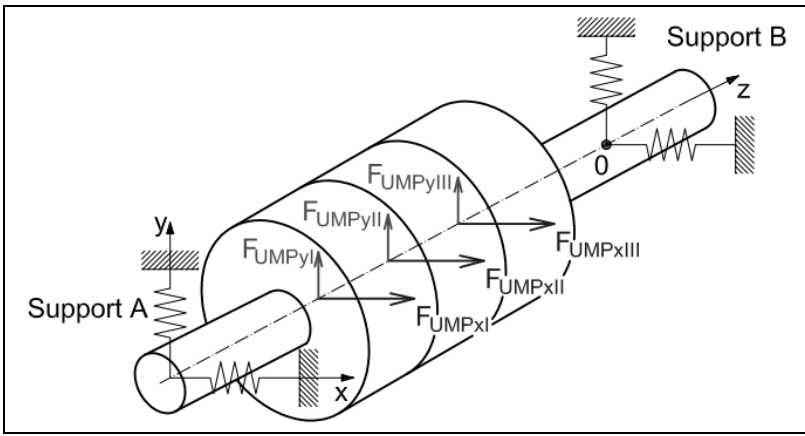

Fig. 3. Rotor of the machine

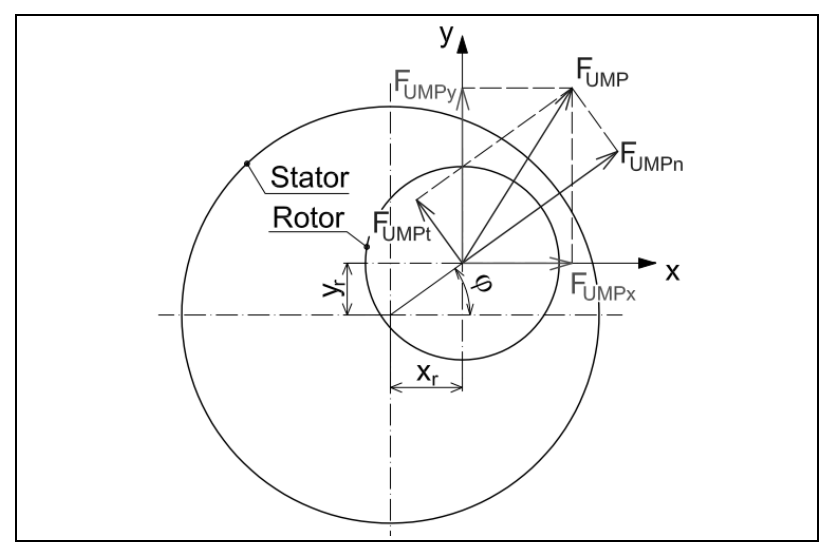

Fig. 4. Calculation of $\mathrm{x}$ and $\mathrm{y}$ components of UMP 


$$
\begin{gathered}
\varphi=\arctan \frac{y_{r}}{x_{r}} \\
{\left[\begin{array}{l}
F_{U M P x} \\
F_{U M P y}
\end{array}\right]=\left[\begin{array}{cc}
\cos \varphi & -\sin \varphi \\
\sin \varphi & \cos \varphi
\end{array}\right] \cdot\left[\begin{array}{l}
F_{U M P n} \\
F_{U M P t}
\end{array}\right]}
\end{gathered}
$$

\section{RESULTS}

The cage induction motor was considered in this paper. It is assumed that the rotor and the stator have ideal cylindrical shape and the rotor is eccentrically mounted due to stator, as seen from Figure 5. Parameters of the studied machine are shown in Table 1.

\begin{tabular}{|l|c|c|}
\hline \multicolumn{1}{|c|}{ Item } & Value & Unit \\
\hline Rated power & 1.1 & $\mathrm{~kW}$ \\
\hline Rated line voltage & 230 & $\mathrm{~V}$ \\
\hline Supply frequency & 50 & $\mathrm{~Hz}$ \\
\hline Rated speed & 2845 & $\mathrm{rpm}$ \\
\hline Number of stator slots & 18 & - \\
\hline Number of rotor slots & 23 & - \\
\hline Number of pole pairs & 1 & - \\
\hline Number of turns of stator coils & 83 & - \\
\hline Stator outer diameter & 62.5 & $\mathrm{~mm}$ \\
\hline Stator inner diameter & 32.25 & $\mathrm{~mm}$ \\
\hline Air gap thickness & 0.25 & $\mathrm{~mm}$ \\
\hline Stator core length & 74 & $\mathrm{~mm}$ \\
\hline Connection of stator coils & star & - \\
\hline Mass of the rotor & 3.4 & $\mathrm{~kg}$ \\
\hline Volume of the rotor & $2.88 \cdot 10^{-4}$ & $\mathrm{~m}$ \\
\hline
\end{tabular}

Tab. 1. Parameters of the studied motor

The dependence of normal and tangential components of UMP on the air gap asymmetry and rotation angle of the rotor bars due to stator windings slots was calculated as first. This Process is described in detail in [5]. Parameter of air gap asymmetry $x_{e x}$ was stepped from 0 by $0.02 \mathrm{~mm}$ to $0.16 \mathrm{~mm}$. For each value of parameter $x_{e x}$, normal and tangential components of UMP were calculated as functions of the rotation angle of the rotor bars due to stator winding slots. Figures 6 - 9 shown examples these dependencies for $x_{e x}=0.02 \mathrm{~mm}$ and $0.12 \mathrm{~mm}$.

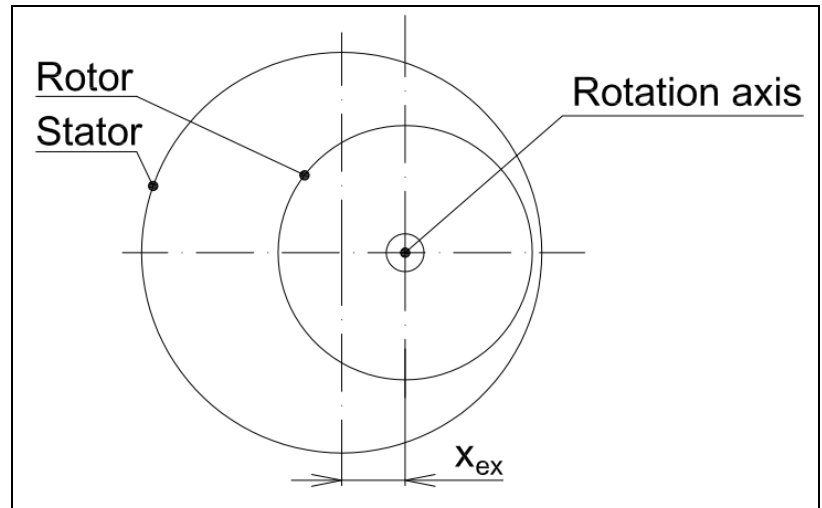

Fig. 5. Type of air gap asymmetry

It can be seen that the amplitudes of $\mathrm{F}_{\mathrm{UMPn}}$ and $\mathrm{F}_{\mathrm{UMPt}}$ is not constant for constant value of parameter $\mathrm{x}_{\mathrm{ex}}$, but oscilate due to slot harmonic effect. This dependence is approximately sinusoidal for small value of parameter $\mathrm{x}_{\mathrm{ex}}$. Sinusoidal behavior of this dependence is disturbed by saturation effect of the ferromagnetic core of the rotor and the stator winding for higher value of parameter $\mathrm{x}_{\mathrm{ex}}$. All obtained results were collect together at the end of this analysis and two two-dimensional arrays which describes the dependence of normal and tangential components of UMP on the air gap asymmetry and rotation angle of the rotor bars due to stator windings slots were drawn up.

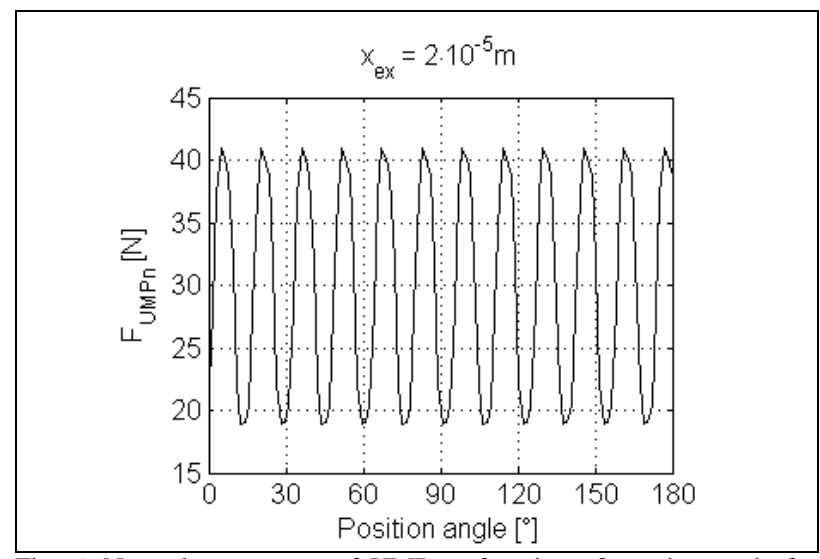

Fig. 6. Normal component of UMP as function of rotation angle for $x_{\text {ex }}=0.02 \mathrm{~mm}$

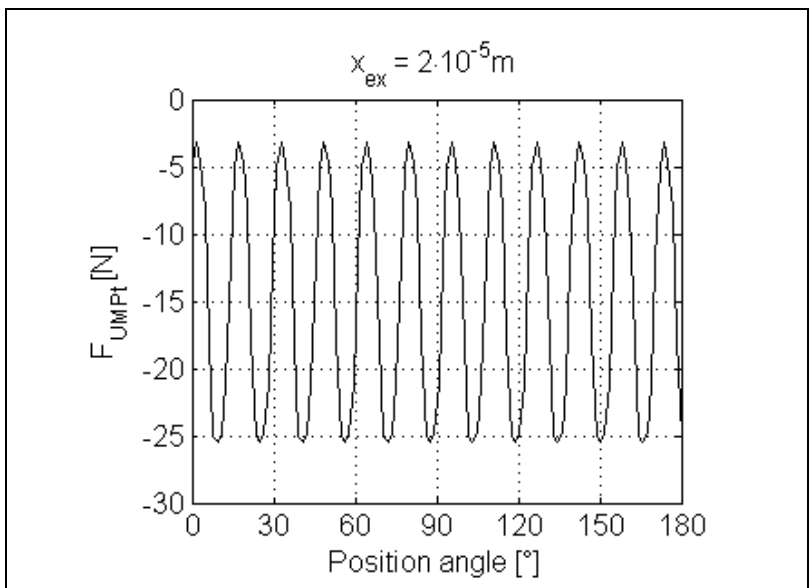

Fig. 7. Tangential component of UMP as function of rotation angle for $x_{e x}=0.02 \mathrm{~mm}$

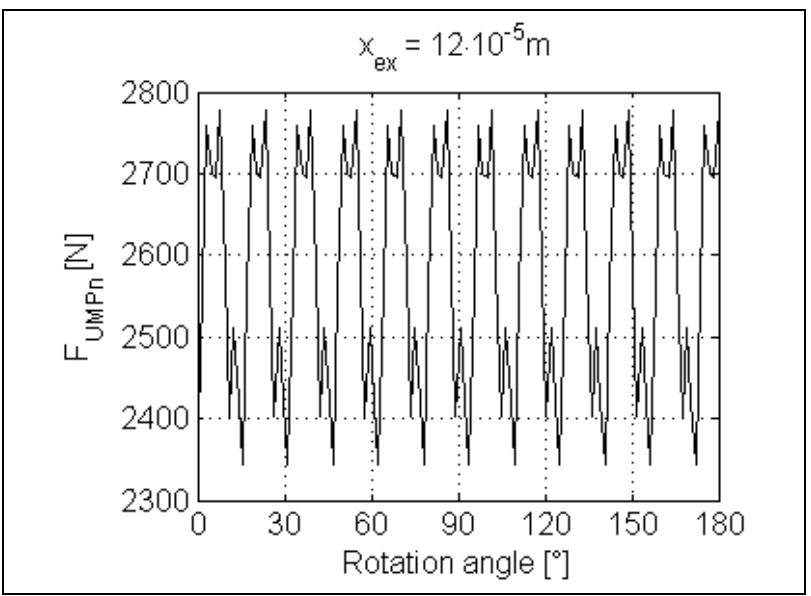

Fig. 8. Normal component of UMP as function of rotation angle for $\mathrm{x}_{\mathrm{ex}}=0.12 \mathrm{~mm}$ 


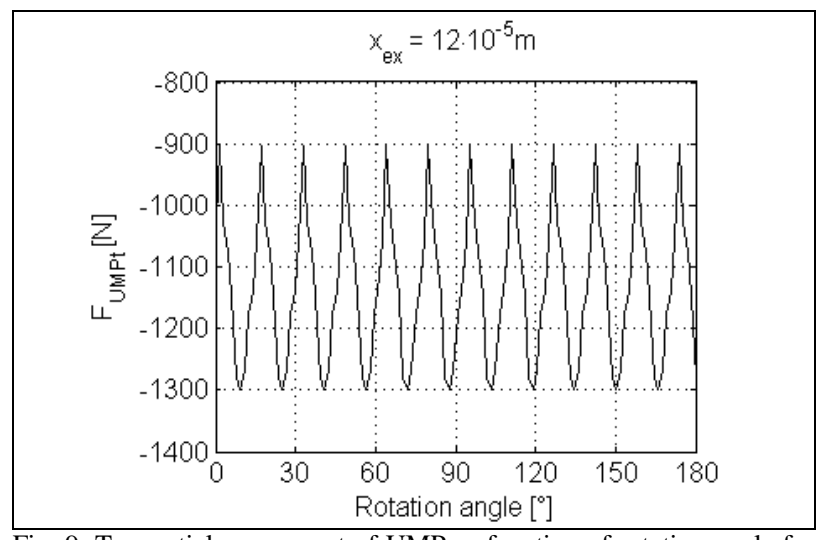

Fig. 9. Tangential component of UMP as function of rotation angle for $x_{e x}=0.12 \mathrm{~mm}$

The dynamic response of the rotor on forced steadystate vibration caused by UMP was calculated in the next step. The calculation had two phases. The static eccentricity grew from zero to the chosen value of $x_{e x}$, at first due to suppression of transient effects. This process took the first five turns. Static eccentricity was constant in the second phase and steady-state response of the rotor on UMP was studied within next ten turns.

The dynamic response of the rotor exited by UMP was studied for three values of parameter $x_{e x}=0.02,0.06$ and $0.12 \mathrm{~mm}$. Rotor of the machine used in this paper is small and thus relatively stiff; therefore influence of the unbalance force caused by dynamic eccentricity of the rotor's center of gravity is neglected. Obtained results are presented as rotor center's orbits in Figures 10,11 and 12. From these Figures, it can be seen that if the parameter of air gap eccentricity $x_{e x}$ is small, the rotor center's orbit has pure oval shape. As the value of $x_{e x}$ increases, the effect of saturation of the stator and rotor winding core affects the magnitude of UMP and thus the dynamic behavior of the rotor of the machine and shape of the rotor center's orbit becomes more complicated. It should be noted that the influence of UMP on dynamic behavior of the rotor is more pronounced for medium and large rotating electrical machines, because these machines have a lower ratio $k / m$, where $k$ is stiffness and $m$ is the mass of the rotor. The calculations are performed for small rotor in this paper, due to experimental verification of obtained results in future.

\section{CONCLUSION}

An efficient way to assess the influence of UMP on dynamic behavior of the rotor of the rotating electric machines, based on the finite element analysis, has been presented. The UMP acting on the rotor was calculated from results of the electromagnetic coupled field analysis by Maxwell stress tensor method. Dependence of UMP on the air gap asymmetry and rotation angle of the rotor bars due to stator winding slots was obtained. The dynamic responses of the rotor on forced steady-state vibration caused by UMP were calculated by finite element time-stepping analysis for different values of parameter of air gap eccentricity.

The next step will be to include the unbalanced force caused by dynamic eccentricity of the rotor's center of gravity to the computational model and to assess its influence on the dynamic response of the rotor.

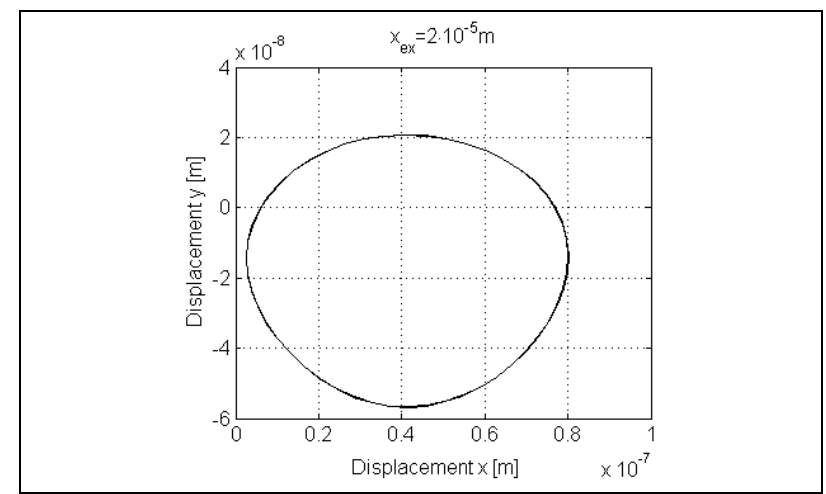

Fig. 11. Rotor center's orbit for $\mathrm{x}_{\mathrm{ex}}=2 \cdot 10^{-5} \mathrm{~m}$

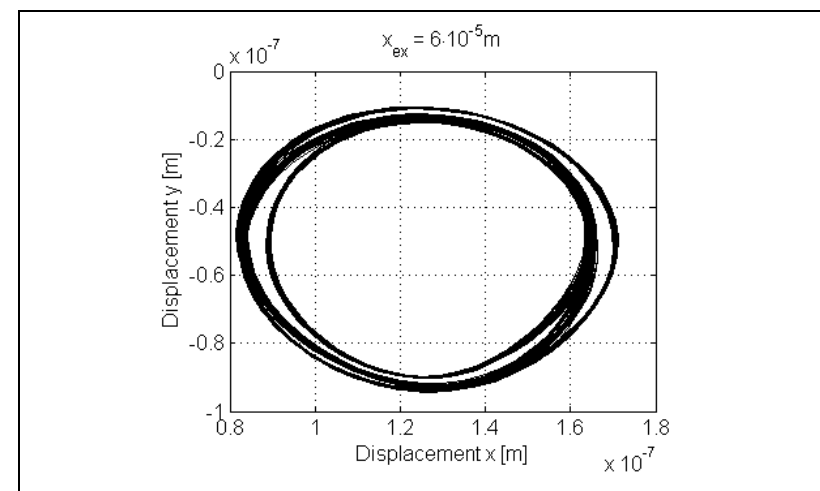

Fig. 11. Rotor center's orbit for $\mathrm{x}_{\mathrm{ex}}=6 \cdot 10^{-5} \mathrm{~m}$

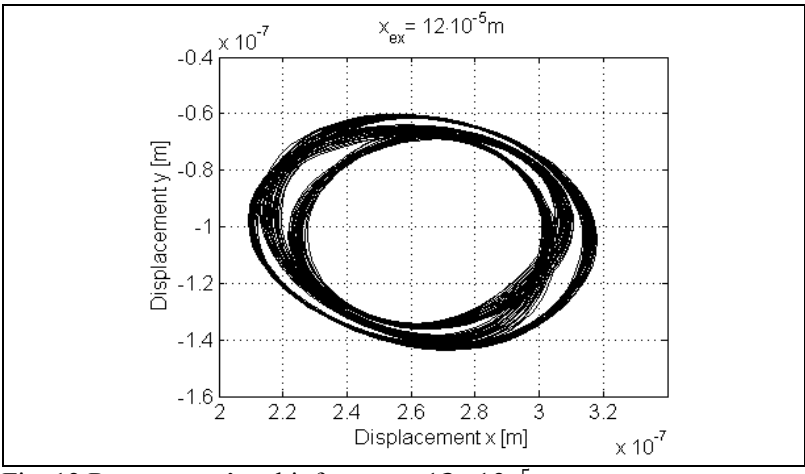

Fig. 12 Rotor center's orbit for $\mathrm{x}_{\mathrm{ex}}=12 \cdot 10^{-5} \mathrm{~m}$

\section{ACKNOWLEDGEMENTS}

The work has been supported by the grant project FSI-S$11-11 / 1190$.

\section{REFERNCES}

[1] Guo, D., Chu, F. \& Chen, D. (2002). The unbalanced magetic pull and its efects on vibration in three-phase generator with eccentric rotor, Journal of Sounds and Vibration, Vol. 254, No. 2, 2002, pp. 297-312

[2] Lundström, N., Aidanpää, J. O. (2010). Dynamics in large generators due to oval rotor and triangual stator shape, Acto Mechanica Sinica, Vol. 27, No. 1, 2010, pp. 18-27

[3] Pennacchi, P. (2008). Computational model for calculating the dynamical behaviour of generators caused by unbalanced magnetic pull and experimental validation, Journal of Sounds and Vibration, Vol. 312, No. 1, 2008, pp. 332-353

4] Holopainen, T. P., Tenhunen, A. \& Arkkio, A. (2002) Electromagnetic circulatory forces and rotordynamic insability in electric machines, Proceedings of the $6^{\text {th }}$ Conference on Rotor Dynamics. Australia, ISBN 0-7344-1963-1, Hahn, E. J. \& Randall, R. B. (Ed.), pp. 446-463, University of New South Wales, Sydney

[5] Donát, M. (2012), Computational modellig of the unbalanced magnetic pull by finite element method, unpubliched 